\title{
Candida albicans forms biofilms on the vaginal mucosa
}

\section{Correspondence \\ M. C. Noverr \\ mnover@lsuhsc.edu}

Received 1 March 2010

Revised 25 July 2010

Accepted 6 August 2010

\author{
M. M. Harriott, ${ }^{1}$ E. A. Lilly, ${ }^{2}$ T. E. Rodriguez, ${ }^{2}$ P. L. Fidel, Jr ${ }^{2,3}$ \\ and M. C. Noverr ${ }^{2,3}$ \\ ${ }^{1}$ Department of Immunology and Microbiology, Wayne State University School of Medicine, \\ Detroit, MI, USA \\ ${ }^{2}$ Department of Oral and Craniofacial Biology, Louisiana State University Health Sciences Center, \\ New Orleans, LA, USA \\ ${ }^{3}$ Department of Microbiology, Immunology, and Parasitology, Louisiana State University Health \\ Sciences Center, New Orleans, LA, USA
}

Current understanding of resistance and susceptibility to vulvovaginal candidiasis challenges existing paradigms of host defence against fungal infection. While abiotic biofilm formation has a clearly established role during systemic Candida infections, it is not known whether C. albicans forms biofilms on the vaginal mucosa and the possible role of biofilms in disease. In vivo and ex vivo murine vaginitis models were employed to examine biofilm formation by scanning electron and confocal microscopy. C. albicans strains included 3153A (lab strain), DAY185 (parental control strain), and mutants defective in morphogenesis and/or biofilm formation in vitro (efg1/ efg 1 and $b c r 1 / b c r 1)$. Both 3153A and DAY815 formed biofilms on the vaginal mucosa in vivo and ex vivo as indicated by high fungal burden and microscopic analysis demonstrating typical biofilm architecture and presence of extracellular matrix (ECM) co-localized with the presence of fungi. In contrast, efg1/efg1 and bcr1/bcr1 mutant strains exhibited weak or no biofilm formation/ECM production in both models compared to wild-type strains and complemented mutants despite comparable colonization levels. These data show for the first time that $C$. albicans forms biofilms in vivo on vaginal epithelium, and that in vivo biotic biofilm formation requires regulators of biofilm formation (BCR1) and morphogenesis (EFG1).

\section{INTRODUCTION}

Vulvovaginal candidiasis (VVC) affects $75 \%$ of all women at least once in their lifetime (Sobel et al., 1998), most often during childbearing years (Sobel, 1992). Another smaller group of women (5-8\%) experience recurrent vulvovaginal candidiasis (RVVC), defined as having three or more episodes per year (Sobel, 1992). Although several Candida species have been implicated in VVC and RVVC, Candida albicans is the predominant aetiological agent, causing 85$95 \%$ of these infections (Sobel et al., 1998).

Current understanding of resistance and susceptibility to VVC challenges existing paradigms of host defence against fungal infection. Although the adaptive immune response plays a major role in protection against oral and gastrointestinal mucosal Candida infections, the innate immune response plays a more prominent role in VVC. In resistant women, vaginal epithelial cells are important for

Abbreviations: CM, confocal microscopy; ConA-TR, concanavalin ATexas red conjugate; ECM, extracellular matrix; (R)VVC, (recurrent) vulvovaginal candidiasis; SEM, scanning electron microscopy. protection against infection (Fidel et al., 2004). In susceptible women, symptomatic VVC is associated with an aggressive neutrophil migration into the vagina and subsequent inflammatory response initiated by the interaction of $C$. albicans with vaginal epithelial cells (Barousse et al., 2001, 2005). This inflammatory response is not protective, but instead appears to be a major cause of the symptoms associated with infection.

Recently there has been tremendous interest in the role of biofilms in infectious diseases. It is estimated that $80 \%$ of human infections result from pathogenic biofilms $(\mathrm{NIH}$, 1999). Biofilms are communities of micro-organisms that are embedded in an extracellular matrix (ECM), forming a complex three-dimensional architecture. Candida biofilm development on abiotic surfaces can be divided into several growth stages, including early, intermediate and mature (Chandra et al., 2001). During early biofilm formation, yeast cells adhere to an appropriate surface and initiate germ tube formation. The intermediate phase is characterized by continued hyphal elongation and production of ECM, which consists of cell wall polysaccharides and protein (Baillie \& Douglas, 2000). Mature biofilms consist 
of a yeast base, with hyphal elements encased in ECM extending away from the surface. Newly formed daughter yeast cells grow out of hyphal elements and are released, seeding new niches for biofilm formation or infection. Moreover, biofilm formation is dependent on the ability to undergo morphogenesis; mutants defective in hyphal formation in vitro are also defective in biofilm formation (Nobile et al., 2006b; Ramage et al., 2002).

Candida biofilms have been studied primarily on abiotic surfaces (reviewed by Blankenship \& Mitchell, 2006; Ramage et al., 2006). Significant attention has been given to Candida biofilm formation of indwelling catheters, which can lead to life-threatening systemic infections (Crump \& Collignon, 2000; Dominic et al., 2007; Kojic \& Darouiche, 2004). More recently, biofilm formation on biotic surfaces has received some attention; C. albicans was shown to form biofilms on oral mucosa in an immunosuppressed murine model of oropharyngeal candidiasis (Dongari-Bagtzoglou et al., 2009). However, in VCC and RVVC it is unknown whether C. albicans exist as biofilms on the vaginal mucosa and, if so, whether the biofilms play a role in the immunopathogenesis.

The purpose of this study was to determine whether Candida forms biofilm on vaginal mucosa, using both an in vivo and a novel ex vivo model. Biofilm-defective $C$. albicans mutants were also used to define specific parameters of biofilm formation associated with the tissue.

\section{METHODS}

Candida albicans strains and handling. C. albicans strains used in this study are listed in Table 1. Wild-type (WT) strains included 3153A (lab strain) and DAY185 (parent strain). Strain DAY185 is a complemented prototroph derived from a triple auxotrophic strain (BWP17; parent, SC5314), which is frequently used to produce knockout and complemented strains of C. albicans. Strains HLC52 (efg1/efg1) and HLC74 (efg1/efg1 + pEFG1) were a gift from G. R. Fink (Whitehead Institute, Cambridge, MA, USA). Strains CJN702 (bcr1/ $b c r 1)$ and CJN698 (bcr1/bcr1 + pBCR1) were a gift from A. P. Mitchell (Carnegie Mellon University, Pittsburgh, PA, USA). For each experiment, strains were subcultured from freezer stocks onto Sabouraud dextrose agar (SDA) plates and incubated at $30{ }^{\circ} \mathrm{C}$ overnight. Cultures were washed in $1 \times$ PBS, counted, and diluted in $1 \times$ PBS for inoculation.

Mouse strains. Female $\mathrm{C} 57 \mathrm{BL} / 6$ or $\mathrm{CBA} / \mathrm{J}$ mice, $6-8$ weeks of age (Charles River and NIH) were used in this study. All mice were maintained at an American Association for the Accreditation of Laboratory Animal Care-accredited animal facility at Wayne State University (WSU) or Louisiana State University Health Sciences Center (LSUHSC) and housed in accordance with the procedures outlined in the Guide for the Care and Use of Laboratory Animals under an animal study proposal approved by the WSU animal investigation committee and the LSUHSC Institutional Animal Care and Use Committee (IACUC).

In vivo model. Mice were administered $0.1 \mathrm{mg}$ 17- $\beta$-oestradiol (Sigma-Aldrich) in $0.1 \mathrm{ml}$ sesame seed oil (Sigma-Aldrich) subcutaneously 3 days prior to inoculation. They were inoculated intravaginally with $20 \mu \mathrm{l}$ C. albicans $3153 \mathrm{~A}\left(2.5 \times 10^{6} \mathrm{ml}^{-1}\right)$, DAY185 $\left(2.5 \times 10^{8} \mathrm{ml}^{-1}\right)$ or biofilm mutants $\left(2.5 \times 10^{8} \mathrm{ml}^{-1}\right)$. Higher inocula were used for DAY185 and mutants because initial experiments demonstrated that $3153 \mathrm{~A}$ colonizes mucosal tissue better than DAY185 and biofilm mutants, as previously reported (Naglik et al., 2008; Taylor et al., 2000). At specified time points, mice were euthanized and their vaginae were excised and cut longitudinally to expose the mucosal surface. The vaginae were then bisected; half the tissue was used to determine fungal load by quantitative plate count and the other half was used for confocal microscopy or scanning electron microscopy.

Ex vivo model. Mice were treated with oestrogen as described above 3 days prior to vaginal harvest. On experimental days, mice were euthanized and their vaginae were excised and cut longitudinally to expose the mucosal surface. The vaginae were placed with the mucosal side facing up in 6-well tissue culture treated plates with $500 \mu \mathrm{l}$ PBS $(1 \times)$, which results in the vaginal mucosal surface being exposed and unsubmerged. Penicillin-streptomycin $(1 \times$, MP Biomedicals) was added to the PBS to prevent growth of normal vaginal bacteria. C. albicans isolates $\left(1 \times 10^{6}\right.$ blastoconidia) were added to the mucosal surface in $0.1 \mathrm{ml} \mathrm{PBS}$ and incubated at $37{ }^{\circ} \mathrm{C}$ with $\mathrm{CO}_{2}$. The inoculum was based on preliminary studies that revealed optimal colonization for microscopic evaluations. Tissues were removed from the incubator at specified time points and bisected but were not washed prior to analysis. Half the tissue was used to determine fungal load and the other half was used for microscopy.

C.f.u. counts. Following harvest, vaginae were placed in $5 \mathrm{ml}$ sterile tubes with $1 \mathrm{ml}$ sterile $\mathrm{H}_{2} \mathrm{O}$. Tissues were weighed and homogenized, and 10-fold dilutions were prepared and plated to SDA with ampicillin and erythromycin to prevent the growth of normal vaginal

Table 1. Strains used in this study

\begin{tabular}{|c|c|c|}
\hline Strain & Genotype & Source \\
\hline $3153 \mathrm{~A}$ & Wild-type & Slutsky et al. (1985) \\
\hline DAY185 & $\begin{array}{l}\text { ura3s : : imm434/ura3s:: imm434 HIS1:: his1:: hisG/his1:: hisG ARG4:: URA3:: arg4:: hisG/ } \\
\text { arg4:: hisG }\end{array}$ & Norice et al. (2007) \\
\hline HLC52 & 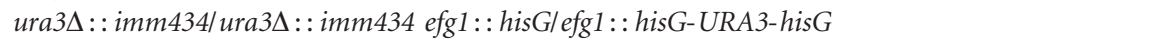 & Lo et al. (1997) \\
\hline HLC74 & ura3s:: imm434/ura3A:: imm434 efg1:: hisG/efg1:: hisG LEU2/LEU2:pEFG1-URA3-LEU2 & Lo et al. (1997) \\
\hline CJN702 & 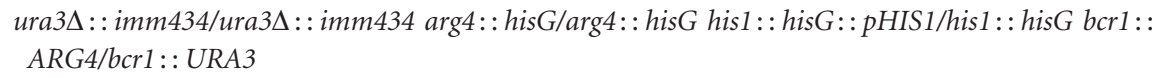 & Nobile \& Mitchell (2005) \\
\hline CJN698 & 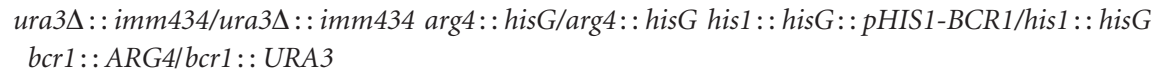 & Nobile \& Mitchell (2005) \\
\hline
\end{tabular}


bacteria. Following an overnight incubation at $37^{\circ} \mathrm{C}$, colonies were counted and $\log$ c.f.u. $\mathrm{ml}^{-1}$ and $\log$ c.f.u. (g tissue) $)^{-1}$ were calculated.

Confocal microscopy (CM). Excised vaginal tissue was placed in 12well dishes and stained with $1 \mathrm{mg} \mathrm{ml}^{-1}$ Calcofluor White (Fluka) to visualize yeast and hyphae and $50 \mu \mathrm{g} \mathrm{ml}^{-1}$ concanavalin A-Texas red conjugate (ConA-TR, Molecular Probes) to stain the biofilm ECM for $20 \mathrm{~min}$ at room temperature. No prior washing or sectioning of tissue was performed. Vaginal tissues were removed from staining wells, gently rinsed with $1 \times$ PBS to remove exogenous stain, and placed epithelial side up onto glass microscope slides. Vaginal tissues were covered with a glass coverslip and slides were examined under oil immersion with a Nikon Eclipse E800 microscope with Metamorph software.

Scanning electron microscopy (SEM). Vaginal tissue was placed in $10 \%$ formalin. Tissues were dehydrated in $20 \%$ ethanol for $1 \mathrm{~min}$, followed by three successive 1 min incubations in $40 \%, 60 \%, 80 \%$, $95 \%$ and $100 \%$ ethanol, and finally by three successive $1 \mathrm{~min}$ incubations in acetone. Dried samples were affixed to SEM stubs and placed into a gold/palladium sputter coater. Specimens were viewed at 300-2000 $\times$ magnification in a Hitachi S-2700 scanning electron microscope (LSUHSC Imaging Core) with the voltage set to $15 \mathrm{kV}$.

Statistical analysis. Statistical analysis of the data was conducted using Prism software (GraphPad). Data were analysed by ANOVA (analysis of variance) followed by Dunnett's multiple comparison test. Significance was defined as $P<0.05$.

\section{RESULTS}

\section{C. albicans forms in vivo and ex vivo biofilms on vaginal mucosae}

To initially determine whether C. albicans forms biofilms on vaginal mucosae, we used both in vivo and ex vivo colonization models using WT strain 3153A. For the in vivo model, vaginal tissue was analysed $48 \mathrm{~h}$ post-inoculation for biofilm formation by scanning election microscopy (SEM) and confocal microscopy (CM). This is a commonly used early time point to monitor colonization during experimental vaginitis. SEM analysis revealed fungal colonization and the presence of a complex network of both yeast and hyphae, and ECM accumulation both in vivo and ex vivo (Fig. 1a, b). CM analysis confirmed that biofilm ECM was present, as demonstrated by the bright red cell-associated staining and the hazy diffuse red material covering the hyphae (Fig. 1d).

We also examined ex vivo biofilm formation on vaginal explants at $48 \mathrm{~h}$ post-inoculation, which is a time point correlating with development of mature biofilm in vitro (Chandra et al., 2001). Ex vivo model biofilms were phenotypically similar to vivo biofilms. At $48 \mathrm{~h}$, a characteristic biofilm was visible by SEM, consisting of yeast and hyphae surrounded by ECM (Fig. 1b). Biofilm ECM was also evident by $\mathrm{CM}$, both cell associated and interspersed between the hyphae (Fig. 1e). No Candida colonization was observed in control uninoculated vaginal epithelium, either in vivo or ex vivo (Fig. $1 \mathrm{c}$, $\mathrm{f}$ and data not shown). It is important to note that although ConA-TR stains epithelial cells, the fluorescence intensity was weaker and exposure time was increased to visualize epithelial cells compared to ConA-TR-stained ECM material (Fig. 1d, e). The presence of c.f.u. following the plating of tissue homogenates under in vivo or ex vivo conditions confirmed the presence of viable C. albicans.

\section{Kinetics of vaginal mucosal biofilm formation}

We next examined the kinetics of biofilm development in both models by SEM, CM and quantitative plate count analysis. Vaginae were harvested at $8,24,48$ and $72 \mathrm{~h}$ postinoculation with $C$. albicans 3153A. Half the tissue was processed for scanning SEM or CM and the other half was used to determine fungal load (c.f.u.). For the in vivo model, yeast had initiated germination on the vaginal epithelium (SEM) by $8 \mathrm{~h}$ post-inoculation, with little or no ECM (CM) (Fig. 2). At $24 \mathrm{~h}$ the initial layer of yeast was evident under the elongating hyphae. Also, a small amount of ECM material was visible, as demonstrated by the diffuse red staining in the CM. At $48 \mathrm{~h}$, the biofilm was more extensive and ECM appeared thicker and covered some of the cellular portion of the biofilmas revealed by SEM and CM (Fig. 2, red staining). By 72 h, ECM accumulation was more uniform by SEM, appearing as a smooth coat on top of the fungal biofilm. By CM, ECM staining appeared cellassociated and also more diffuse, lying above the cellular portion of the biofilm. The pattern of colonization with the biofilm was not confluent over all of the vaginal tissue, but did form patches of thicker and thinner biofilms spread over the tissue (data not shown). Fungal burden also increased steadily with time, starting at $\approx 10^{3}$ c.f.u. $(\mathrm{g} \text { tissue })^{-1}$ at $2 \mathrm{~h}$ and increasing to $\approx 10^{5}$ c.f.u. (g tissue $)^{-1}$ by $72 \mathrm{~h}$ (Fig. 3a). Maximal fungal burden was observed by $72 \mathrm{~h}$; however, very little change was observed between 48 and $72 \mathrm{~h}$.

For the ex vivo model, considerable yeast had germinated on the vaginal mucosa by $8 \mathrm{~h}$, with little ECM present $(\mathrm{CM})$ and the mucosal surface could be seen under the hyphae (SEM) (Fig. 2). At $24 \mathrm{~h}$, a hyphal network was formed (SEM) and biofilm ECM was evident (CM, red staining). By $48 \mathrm{~h}$, the biofilm was significantly denser than at $24 \mathrm{~h}$ and ECM appeared more prevalent (SEM). At $72 \mathrm{~h}$, yeast forms $(\mathrm{CM})$ were evident at the apical surface of the biofilm in conjunction with the residential hyphae, which coincided with the predicted dispersal phase of biofilm growth (Blankenship \& Mitchell, 2006). As with in vivo biofilms, ex vivo biofilm fungal burden steadily increased over time, starting at $\approx 10^{4}$ c.f.u. (g tissue) $)^{-1}$ at $2 \mathrm{~h}$ and increasing to $\approx 10^{8}$ c.f.u. (g tissue) ${ }^{-1}$ by $48-72 \mathrm{~h}$ (Fig. $3 \mathrm{~b}$ ). Maximal fungal burden was observed by $48 \mathrm{~h}$ and c.f.u. remained relatively unchanged up to $72 \mathrm{~h}$.

\section{Role of morphogenesis regulator EFG1 and biofilm regulator BCR1}

To determine requirements for biofilm formation on vaginal tissue and whether biofilm formation was required for vaginal colonization, we evaluated C. albicans mutant 


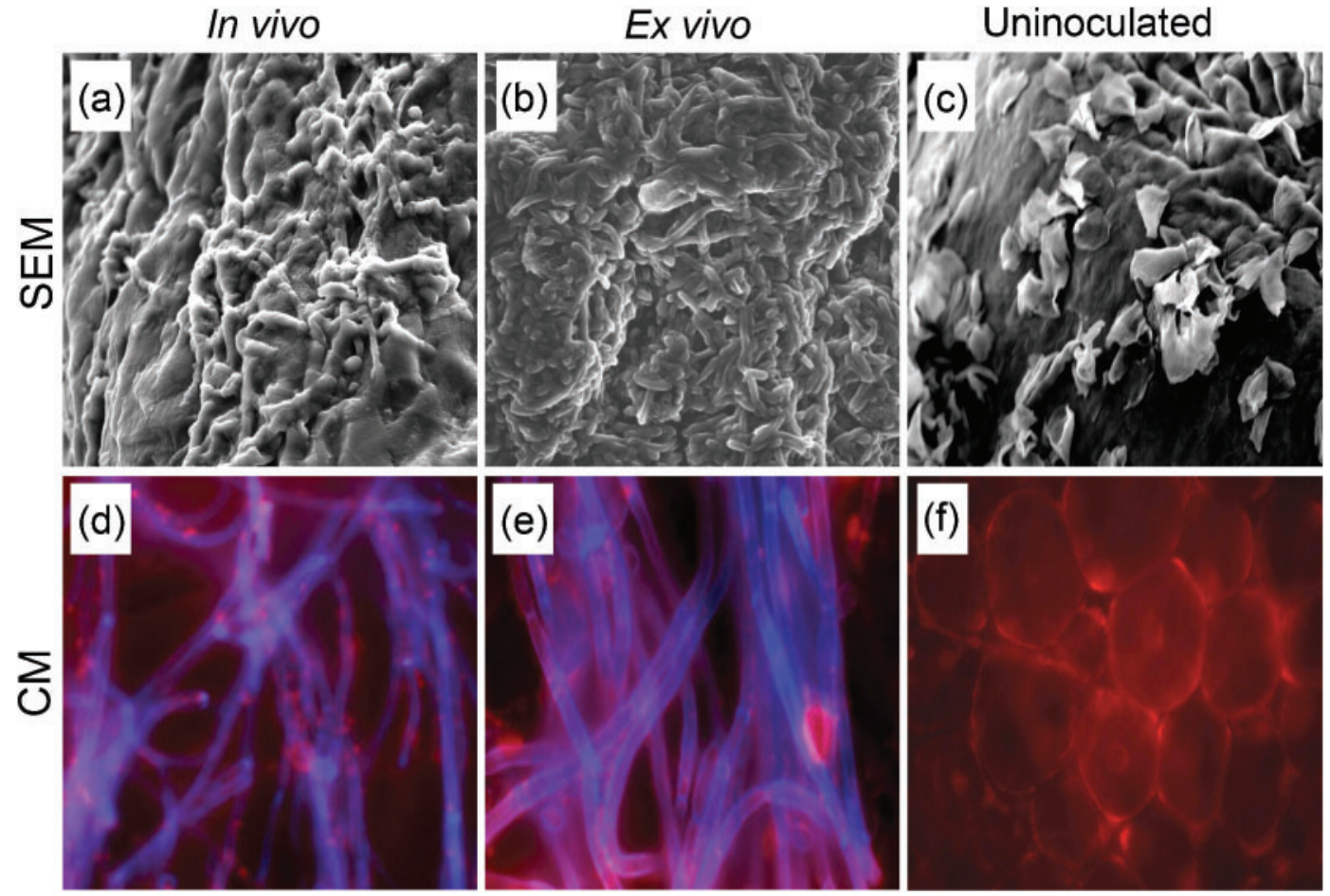

Fig. 1. Establishment of vaginal mucosal biofilm. $(a, d)$ For the in vivo model, mice were administered oestrogen and intravaginally inoculated with C. albicans $3153 \mathrm{~A}\left(5 \times 10^{4}\right.$ blastoconidia). At $48 \mathrm{~h}$ post-inoculation, vaginae were excised and the tissue was processed for SEM or CM. For CM, vaginae were stained with calcoflour white (blue) to visualize yeast/hyphae, and with ConATR to visualize biofilm ECM. (b, e) For the ex vivo model, mice were administered oestrogen and vaginae were harvested at $48 \mathrm{~h}$ post-inoculation. Tissues were inoculated with C. albicans $3153 \mathrm{~A}\left(10^{6}\right.$ blastoconidia). At $48 \mathrm{~h}$ post-inoculation, vaginae were bisected and processed for SEM or CM and confirmation of fungal burden. (c, f) In vivo control tissues were uninoculated. SEM images were taken at $1000 \times$ magnification, and CM images at $600 \times$ magnification. The figure shows representative images of areas of biofilm growth from three independent repeats; $n=4$ mice or vaginal explants per time point.

strains with deletions in transcriptional regulators of morphogenesis (efg1/efg1) and biofilm formation (bcrl/ bcr1) and complemented mutants in the in vivo and ex vivo models. These mutants have previously been shown to form poor or no biofilms on abiotic surfaces (Nobile et al., 2006a, b; Nobile \& Mitchell, 2005; Ramage et al., 2002). For the in vivo model, we chose to examine biofilm mutants at $72 \mathrm{~h}$, the time at which mature biofilms are evident with wild-type strains. Microscopic evaluation demonstrated that in vivo biofilms of strain DAY185 (parental control strain) were similar in appearance to 3153A biofilms at $72 \mathrm{~h}$ (Fig. 4a). Similar results were observed with the wildtype strain SC5314, which is the parent of control strain DAY185 (data not shown). This also confirms that the URA3 marker did not affect vaginal colonization levels, as SC5314 contains URA3 at the native locus and DAY185 contains URA3 at a non-native locus. Yeast and hyphae were present and ECM was evident, as indicated by the smooth covering overlying the cells in the SEM image and diffuse red (ConA-TR) staining overlying the cells in the CM image. In contrast, the efgl/efg1 mutant produced only yeast and pseudohyphal forms, and no ECM was detected by $\mathrm{CM}$, as indicated by the lack of diffuse ConA-TR staining, although staining of underlying epithelial cells was evident. The efg1/efg1 mutant was not detectable by SEM, possibly due to the inability of this strain to remain attached to the epithelium during the tissue-processing procedure. Biofilm formation was restored in the complemented strain efg1/efg1 $+\mathrm{p} E F G 1$ to levels similar to those of the WT strain. Despite the lack of biofilm formation in vivo, the efg1/efg1 mutant strain exhibited colonization levels that were not significantly different from the parental control strain or the complemented mutant (Fig. 4b).

$B C R 1$ is a transcriptional regulator that is required for biofilm formation on abiotic surfaces in vitro and in vivo, forming thin weakly adherent biofilms composed of yeast and hyphae, but with little ECM production (Nobile et al., 2006a; Nobile \& Mitchell, 2005). During vaginal in vivo biofilm formation the $b c r 1 / b c r 1$ mutant was also able to produce some hyphae but very little ECM was visible by SEM, which is evident by the lack of smoothness and the clarity of the cell shape. The ability to form a vigorous biofilm with substantial ECM production was restored in the complemented mutant $(b c r 1 / b c r 1+\mathrm{p} B C R 1)$. In fact 

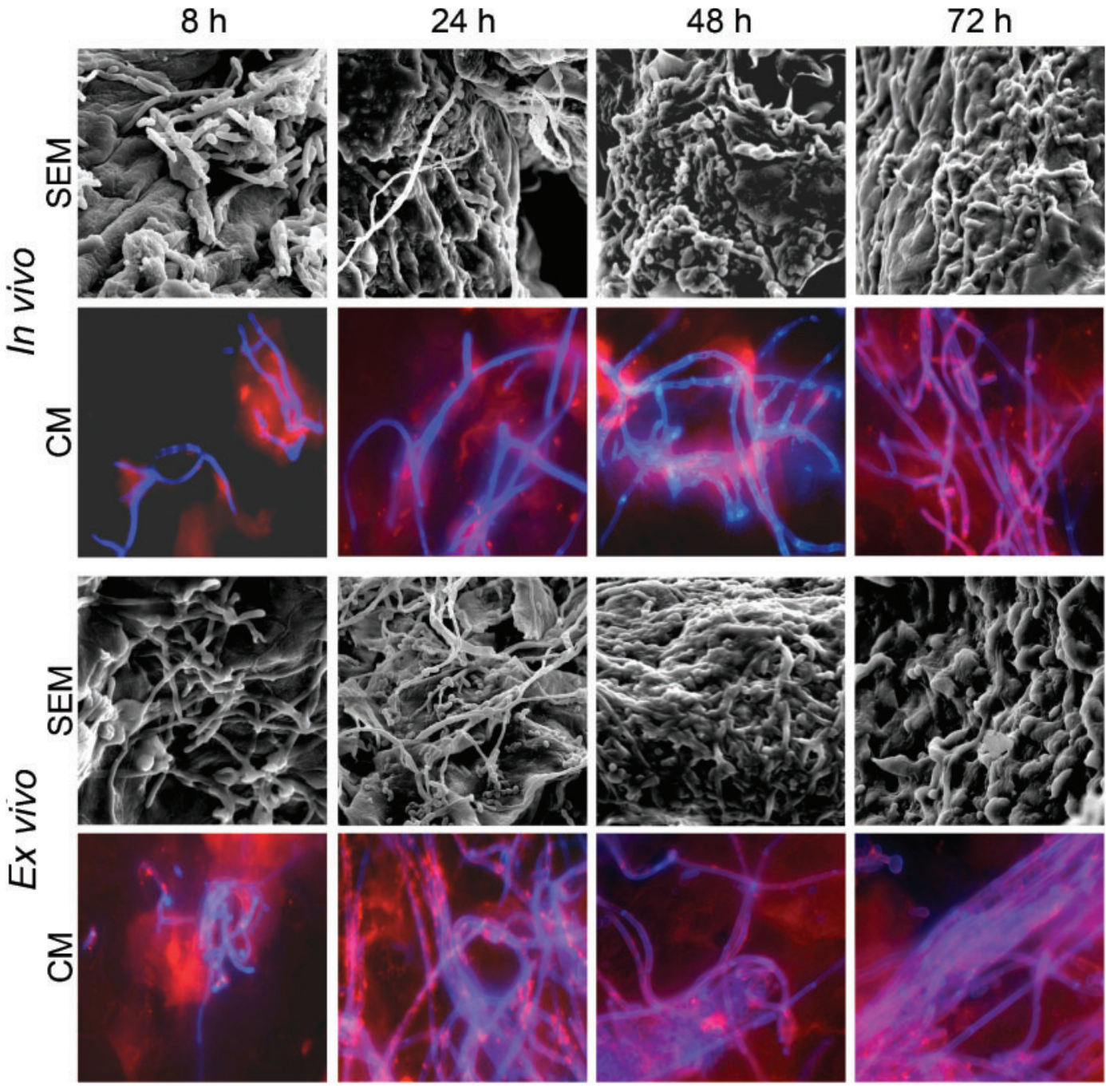

Fig. 2. Microscopic analysis of vaginal mucosal biofilm formation in vivo and ex vivo. For the in vivo model, mice were administered oestrogen and intravaginally inoculated with C. albicans $3153 \mathrm{~A}\left(5 \times 10^{4}\right.$ blastoconidia), and vaginae were harvested at 8, 24, 48 and $72 \mathrm{~h}$. For the ex vivo model, mice were administered oestrogen and vaginae were harvested and inoculated with C. albicans $3153 \mathrm{~A}\left(10^{6}\right.$ blastoconidia) and incubated for $8,24,48$, or $72 \mathrm{~h}$. Tissues were bisected and processed for SEM, CM or fungal burden (Fig. 3). SEM images were taken at 1000× magnification, and CM images at $600 \times$ magnification. The figure shows representative images of areas of biofilm growth from three independent repeats, $n=4$ mice or vaginal explants per time point.

both CM and SEM showed apparently increased ECM production by the complemented strain. Again, there was no significant difference in colonization levels with the $b c r 1 / b c r 1$ mutant strain compared with the parental and complemented strains (Fig. 4b).

For the ex vivo model, oestrogenized vaginal explants were inoculated with the same isolates and incubated for $48 \mathrm{~h}$, the earliest time point for mature biofilm formation by wild-type strains. Microscopic examination demonstrated that the WT parent strain DAY185 formed biofilms ex vivo similar to 3153A (Fig. 5a). Similar results were observed with the wild-type strain SC5314, which is the parent of control strain DAY185 (data not shown). An extensive hyphal network was present with significant ECM, as indicated by the smooth covering overlying the cells in the SEM image and diffuse red staining (ConA-TR) overlying the calcofluor-white-stained fungal cells in the CM image. The efgl/efg1 mutant colonized and produced yeast and pseudohyphal forms at $48 \mathrm{~h}$ with no ECM present (SEM). In contrast to DAY185, there was a lack of diffuse ConATR staining with the efg1/efg 1 mutant by $\mathrm{CM}$, indicating a lack of ECM. Similar to the in vivo model, very few organisms were visible by SEM. Interestingly, the complemented mutant was unable to produce significant amounts of hyphae ex vivo, but still was able to produce ECM visible by CM and SEM. The bcrl/bcrl mutant produced both yeast and a few hyphae, but no ECM was 


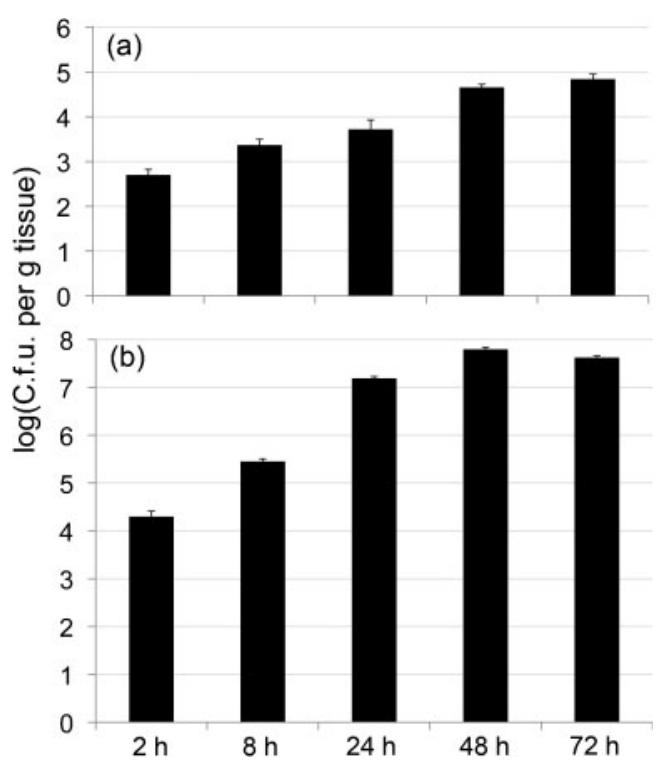

Fig. 3. In vivo and ex vivo kinetics of vaginal fungal burden. For the in vivo model (a), mice were administered oestrogen and intravaginally inoculated with C. albicans $3153 \mathrm{~A}\left(5 \times 10^{4}\right.$ blastoconidia), and vaginae were harvested at $8,24,48$ and $74 \mathrm{~h}$. For the ex vivo model (b), mice were administered oestrogen and vaginae were harvested and inoculated with C. albicans $3153 \mathrm{~A}$ $\left(10^{6}\right.$ blastoconidia) and incubated for $8,24,48$, or $72 \mathrm{~h}$. Tissues were bisected and processed for quantitative plate counts. The same mice were used for microscopy (Fig. 2) and c.f.u. analysis. Results represent means ( $\pm \mathrm{SE}$ ) of three independent repeats; $n=4$ mice or vaginal explants per time point.

visible by SEM. The same result was also evident by CM, which showed a lack of diffuse ConA-TR staining, although underlying lightly stained epithelial cells could be seen (Fig. 5a). In contrast, ECM production was restored in the complemented mutant. However, no differences in colonization were observed between WT strain DAY185, mutants, or complemented mutants (Fig. 5b).

\section{DISCUSSION}

Biofilms are present ubiquitously and have established a unique niche on abiotic and biotic surfaces. To our knowledge this is the first report of an in vivo fungal mucosal biofilm in an immunocompetent animal model of vaginitis. Prior to this study, the only in vivo vaginal biofilm characterized was bacterial, consisting of Gardnerella vaginalis (Scott et al., 1989; Swidsinski et al., 2008; van der Meijden et al., 1988). In this study, we demonstrate by microscopic analysis that $C$. albicans biofilms are present on the vaginal mucosa. C. albicans vaginal biofilms have biofilm architecture typical of in vitro-grown C. albicans biofilms, consisting of yeast and hyphae forming a complex network surrounded by ECM (Chandra et al., 2001; Ramage et al., 2001). ECM was evident by SEM, appearing as a thick film covering the cellular portion of the biofilm. For CM analysis, we used ConA-TR staining to visualize ECM. ConA is a lectin that binds to C. albicans polysaccharides secreted as part of the matrix. $\beta$-Glucan is a cell wall polysaccharide that has been detected in Candida ECM by a Limulus amoebocyte lysate clotting enzyme based assay (Nett et al., 2007a, b). However, a monoclonal $\beta$-glucan antibody, which stains the cell wall or cell-associated matrix, was unable to stain the non-cell-associated ECM that is interspersed between the cells (Dongari-Bagtzoglou et al., 2009). In this same study, ConA staining effectively stained the interspersed $\mathrm{ECM}$, and it has been used by many investigators to visualize Candida biofilm matrix (Chandra et al., 2001; Dongari-Bagtzoglou et al., 2009; Nobile \& Mitchell, 2005). Therefore, our observations satisfy the definition of a biofilm and provide evidence for the presence of biofilm on the vaginal mucosa. In addition to the in vivo model, we developed an ex vivo model using vaginal explants, which were kept in a moist controlled environment. Although no exogenous nutrients were provided, C. albicans was able to adhere, and to form hyphae and subsequent biofilms encased in ECM on vaginal explants. The ability to form a biofilm in the absence of exogenous growth media demonstrates that growth occurs most likely by scavenging host nutrients.

Kinetics studies showed that in vivo and ex vivo biofilm formation followed closely with increases in fungal burden. Although the c.f.u. assay may under-represent the actual cell numbers, due to the presence of hyphae in the biofilm, we obtained consistent measurements using this method. One caveat is that testing mutants with defects in morphogenesis could affect the c.f.u. levels relative to a wild-type strain. However, systemic infection models routinely use the c.f.u. assay to assess tissue fungal burden in vivo. One way to address this is to use an alternative assay for fungal burden such as a chitin assay to confirm results from the c.f.u. assay. Our results are also consistent with the kinetics of in vitro biofilms grown on silicone discs, which show steady increases in dry weight measurements until 36-48 $\mathrm{h}$ and then remain stationary (Chandra et al., 2001; Hawser \& Douglas, 1994). In addition, the SEM and CM results for both models progressively showed evidence of ECM. The in vivo and ex vivo vaginal biofilm development phases closely resemble those of in vitro biofilms (Chandra et al., 2001; Ramage et al., 2001). In vitro biofilms on abiotic surfaces have distinct developmental phases: the early phase $(\approx 0-11 \mathrm{~h})$, in which adhesion and morphogenesis occurs; the intermediate phase $(\approx 12-$ $30 \mathrm{~h}$ ), in which the hyphae continue growth and ECM begins to emerge; and the maturation phase $(\approx 38-72 \mathrm{~h})$, by which ECM completely surrounds the biofilm. Interestingly, the ex vivo model had an accelerated pace compared to the in vivo model, which could be due to several factors. First, the ability of the whole intact tissue in vivo to mount an antifungal response could play a role in limiting fungal growth. Second, in vivo vaginal tissue 
(a)
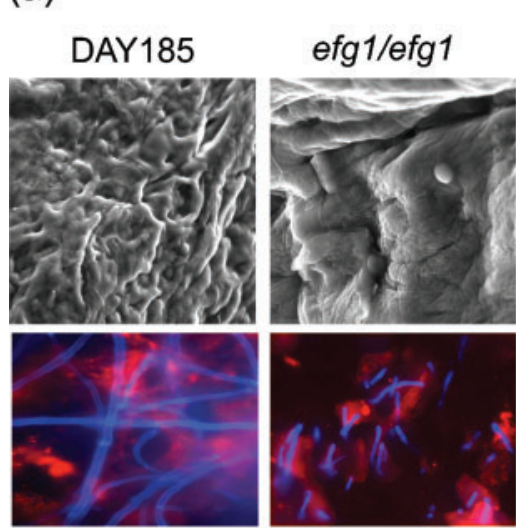

(b)
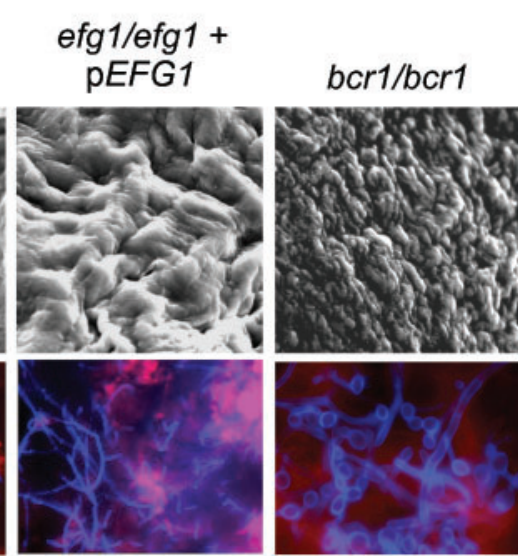

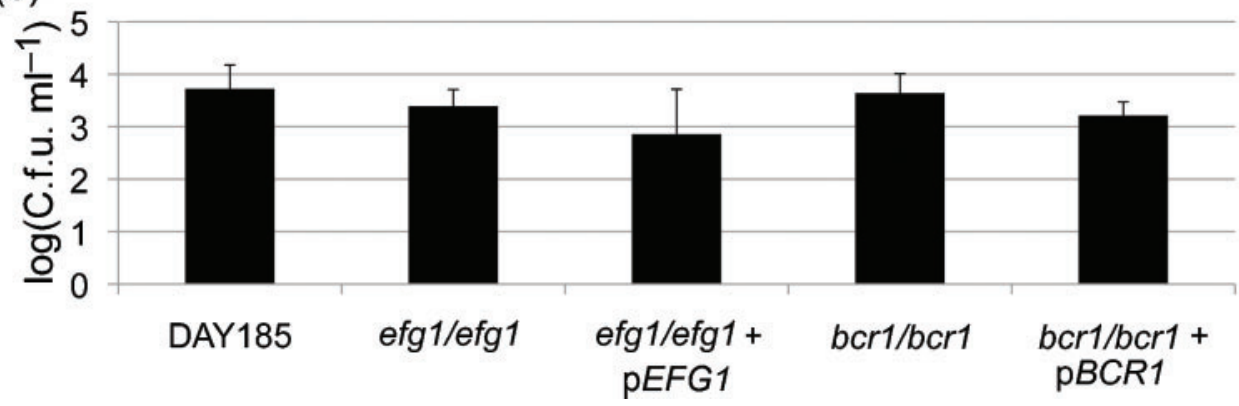

Fig. 4. Role of EFG1 and $B C R 1$ during in vivo vaginal biofilm formation. Mice were administered oestrogen and intravaginally inoculated with C. albicans strain DAY185, efg1/efg1, efg1/efg1+pEFG1, bcr1/bcr1 or bcr1/bcr1+pBCR1 $\left(5 \times 10^{6}\right.$ blastoconidia), and vaginae were harvested at $72 \mathrm{~h}$. Tissues were bisected and processed for (a) SEM or CM, or (b) quantitative plate counts. The figure shows representative images or means ( \pm SE) of three independent repeats; $n=4$ mice or vaginal explants per time point.

contains many secretions, composed of electrolytes, organic acids and proteins, which maintain properties such as pH and osmolarity (Owen \& Katz, 1999). Finally, ex vivo in a tissue-culture setting, the vaginal tissue is completely exposed and unsubmerged in PBS containing antibiotics, whereas intact tissue has a degree of protection by bacterial microbiota.

To further evaluate $C$. albicans vaginal biofilms, we tested several biofilm-deficient mutants in the in vivo and ex vivo murine models. EFG1 encodes a transcriptional factor that is required for morphogenesis under most hypha-inducing conditions in vitro (Lo et al., 1997; Stoldt et al., 1997). Accordingly, the efgl/efgl mutant is unable to form biofilms in vitro in RPMI or YNB + glucose; however, its biofilm formation ability in vivo has not been previously reported (Ramage et al., 2002; Watamoto et al., 2009). In both our in vivo and ex vivo vaginal biofilm models, the efg1/efg1 mutant is also defective in morphogenesis and biofilm formation, lacking typical biofilm architecture and ECM production. This observation is in agreement with previous reports that EFG1 is required for production of hyphae and subsequent biofilm formation in vitro (Ramage et al., 2002). Interestingly, few or no organisms were detectable by SEM, which requires significant sample processing with several dehydration procedures. This observation suggests that although colonization is detectable by c.f.u. analysis, these strains exhibit defects in adhesion, and are unable to remain adherent throughout the SEM processing procedure. The complemented mutant was able to form a normal biofilm consisting of yeast and hyphae and substantial ECM production in vivo similar to WT. However, ex vivo, this strain was unable to produce hyphae, forming a yeast-dominated biofilm with considerable ECM. One explanation for this observation is that morphogenesis-inducing signals could be stronger in vivo than ex vivo. Another possibility is that the differences in microenvironment that result from the way the explants were incubated (unsubmerged and exposed to $\mathrm{CO}_{2}$ ) could influence the growth of the mutants. This could lead to differential EFG1 expression patterns in the WT strain compared with the complemented strain ex vivo. This is important when considering appropriate models for the future, as well as defining what the actual signals are in vivo which lead to biofilm formation. Although this remains to be proven, these results also imply that Efg1 acts on two (separable) levels during biofilm formation, i.e. ECM production and hyphal growth. 
(a)
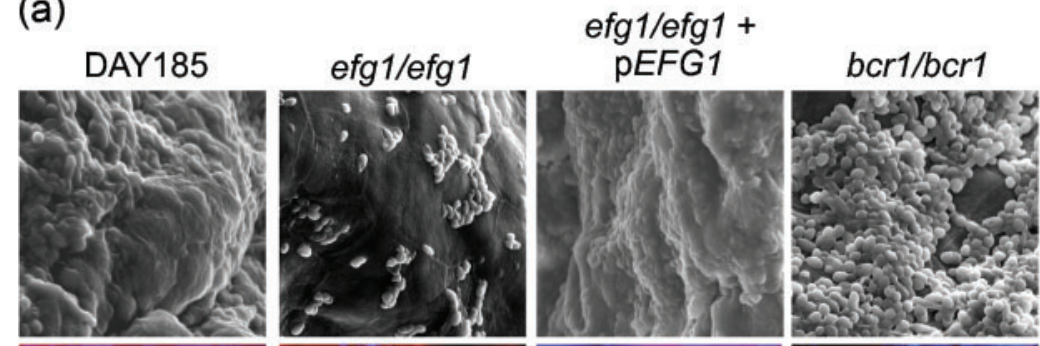

$b c r 1 / b c r 1+$
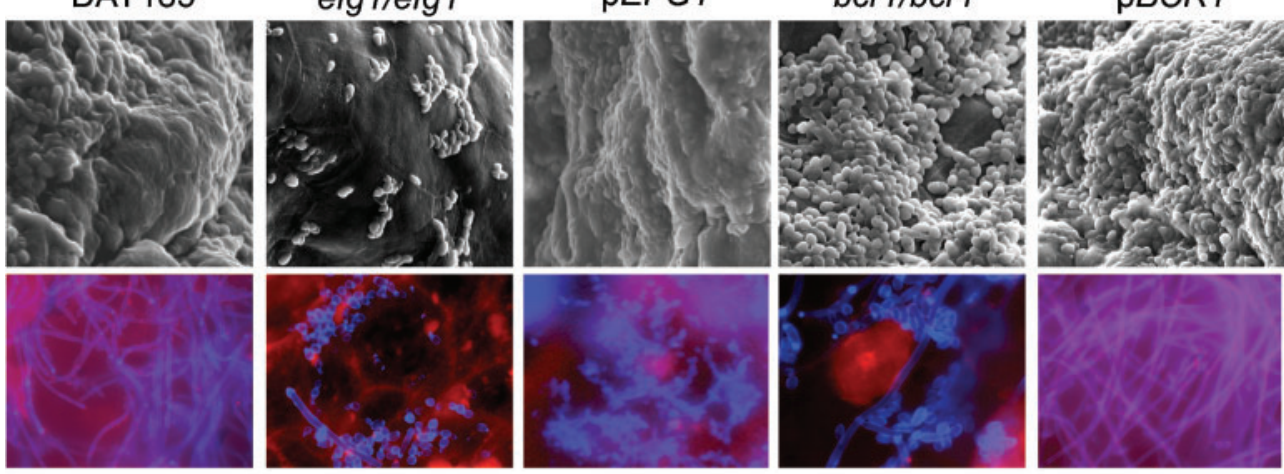

(b)

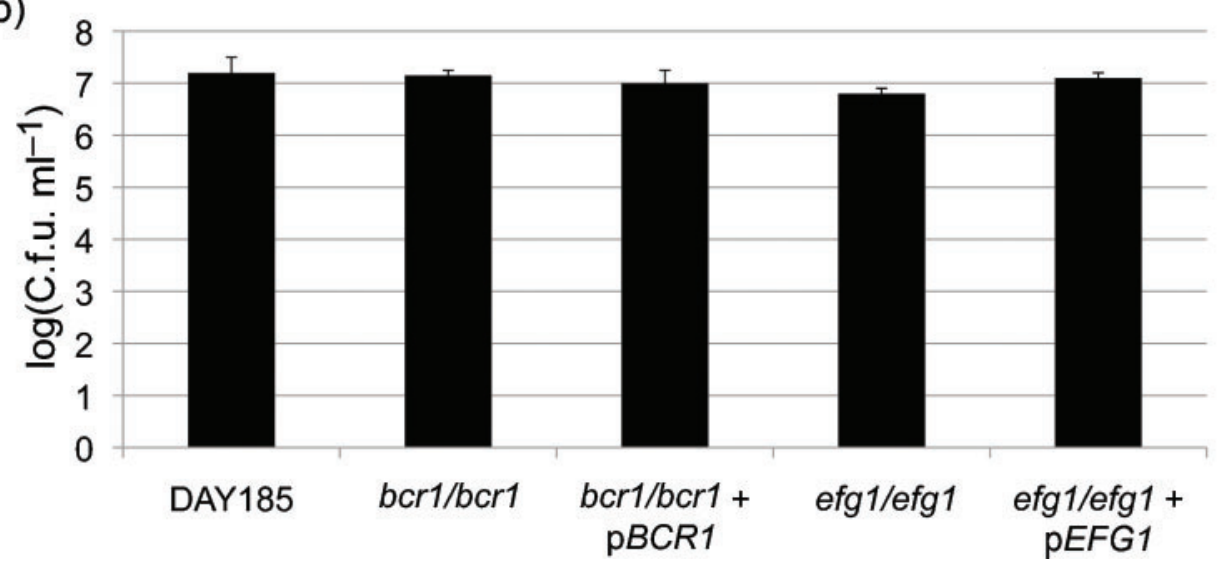

Fig. 5. Role of $E F G 1$ and $B C R 1$ during ex vivo vaginal biofilm formation. Mice were administered oestrogen and vaginae were harvested and inoculated with C. albicans strains DAY185, efg1/efg1, efg1/efg $1+\mathrm{p} E F G 1$, bcr $1 / \mathrm{bcr} 1, \operatorname{bcr} 1 / \mathrm{bcr} 1+\mathrm{p} B C R 1$ $\left(10^{6}\right.$ blastoconidia) and incubated for $48 \mathrm{~h}$. Tissues were bisected and processed for (a) SEM or CM, or (b) quantitative plate counts. The figure shows representative images or means $( \pm \mathrm{SE})$ of three independent repeats; $n=4$ mice or vaginal explants per time point.

$B C R 1$ encodes a transcriptional regulator that is required for production of vigorous biofilms on abiotic surfaces in vitro and in vivo in a catheter model (Nobile et al., 2006a; Nobile \& Mitchell, 2005). The defect in biofilm formation is likely due to a lack of proper adhesin expression on the cell surface, but not due to a defect in morphogenesis (Nobile et al., 2006a). In our vaginal models, the $b c r 1 / b c r 1$ mutant was defective in biofilm formation both in vivo and ex vivo, producing little or no ECM. However, the ability to form mature biofilms in both models was restored in the complemented strain. These results are in agreement with a previous study that demonstrated that BCR1 is necessary for normal biofilm development in vitro and in vivo in a rat catheter model (Nobile et al., 2006a, b). One notable difference is that the $b c r 1 / b c r 1$ mutant formed more hyphae and ECM under in vivo conditions compared with ex vivo conditions. This again indicates that there may be stronger morphogenesis-inducing signals in vivo, which could be due to differences in the microenvironment resulting from the incubation method for the explants.
Another issue is that while the efgl/efg1 mutant was unable to remain attached for SEM visualization, the $b c r 1 / b c r 1$ mutant was more adherent. Efg1 is upstream of Bcr1 in relation to the regulation of genes involved in adherence and biofilm formation. Therefore, the efgl/efgl mutant has additional defects, including defects in morphogenesis. While the adhesion of these mutants has not been directly compared on vaginal tissues, it is possible that the Efg1 regulates additional adhesins not regulated by Bcr1. Another possible explanation is that these mutants could result in differential induction of epithelial surface proteins that are adhesin targets. Overall, the fact that the mutants that are deficient in biofilm formation in vitro are also deficient in biofilm formation in vivo and ex vivo on vaginal mucosa supports the assertion that $C$. albicans forms true biofilms in our models.

This first report of C. albicans biofilm formation on vaginal mucosal tissue is extremely relevant as a newly characterized biotic surface Candida biofilm in an immunocompetent 
host. It is unknown if Candida always exists as a mature biofilm on mucosal surfaces, or only during times of increased fungal burden (e.g. exacerbation of the symptomatic condition during infection). This raises a series of interesting questions: does the presence of a biofilm determine whether $C$. albicans behaves as a pathogen, and does the biofilm play a role in the host response and/or pathogenesis of disease? Another unknown is how commensal vaginal bacteria influence $C$. albicans mucosal biofilm formation. Finally, with C. albicans rarely exhibiting resistance to azoles during VVC (Bauters et al., 2002; Mathema et al., 2001), how does this relate to the high rates of biofilm resistance on abiotic surfaces? The in vivo and novel ex vivo murine models employing biofilm-defective mutants will be excellent to investigate these important questions.

\section{ACKNOWLEDGEMENTS}

This work was supported by National Institutes of Health grants R01 AI72406-01 (M.C.N., M.M.H.) and R01 AI32556 (P.L.F., E.A.L.) and, in part, by the Louisiana Vaccine Center and the South Louisiana Institute for Infectious Disease Research sponsored by the Louisiana Board of Regents. We wish to thank our Candida community colleagues for generous donation of mutant strains: Dr Gerald Fink (Whitehaead Institute) for strains HLC52 and HLC74; and Dr Aaron Mitchell (Carnegie Mellon University) for strains DAY185, CJN702 and CJN698.

\section{REFERENCES}

Baillie, G. S. \& Douglas, L. J. (2000). Matrix polymers of Candida biofilms and their possible role in biofilm resistance to antifungal agents. J Antimicrob Chemother 46, 397-403.

Barousse, M. M., Steele, C., Dunlap, K., Espinosa, T., Boikov, D., Sobel, J. D. \& Fidel, P. L., Jr (2001). Growth inhibition of Candida albicans by human vaginal epithelial cells. J Infect Dis 184, 14891493.

Barousse, M. M., Espinosa, T., Dunlap, K. \& Fidel, P. L., Jr (2005). Vaginal epithelial cell anti-Candida albicans activity is associated with protection against symptomatic vaginal candidiasis. Infect Immun $\mathbf{7 3}$ 7765-7767.

Bauters, T. G., Dhont, M. A., Temmerman, M. I. \& Nelis, H. J. (2002). Prevalence of vulvovaginal candidiasis and susceptibility to fluconazole in women. Am J Obstet Gynecol 187, 569-574.

Blankenship, J. R. \& Mitchell, A. P. (2006). How to build a biofilm: a fungal perspective. Curr Opin Microbiol 9, 588-594.

Chandra, J., Kuhn, D. M., Mukherjee, P. K., Hoyer, L. L., McCormick, T. \& Ghannoum, M. A. (2001). Biofilm formation by the fungal pathogen Candida albicans: development, architecture, and drug resistance. J Bacteriol 183, 5385-5394.

Crump, J. A. \& Collignon, P. J. (2000). Intravascular catheterassociated infections. Eur J Clin Microbiol Infect Dis 19, 1-8.

Davis, D., Edwards, J. E., Jr, Mitchell, A. P. \& Ibrahim, A. S. (2000). Candida albicans RIM101 pH response pathway is required for hostpathogen interactions. Infect Immun 68, 5953-5959.

Dominic, R. M., Shenoy, S. \& Baliga, S. (2007). Candida biofilms in medical devices: evolving trends. Kathmandu Univ Med J (KUMJ) 5, 431-436.
Dongari-Bagtzoglou, A., Kashleva, H., Dwivedi, P., Diaz, P. \& Vasilakos, J. (2009). Characterization of mucosal Candida albicans biofilms. PLoS ONE 4, e7967.

Fidel, P. L., Jr, Barousse, M., Espinosa, T., Ficarra, M., Sturtevant, J., Martin, D. H., Quayle, A. J. \& Dunlap, K. (2004). An intravaginal live Candida challenge in humans leads to new hypotheses for the immunopathogenesis of vulvovaginal candidiasis. Infect Immun 72, 2939-2946.

Hawser, S. P. \& Douglas, L. J. (1994). Biofilm formation by Candida species on the surface of catheter materials in vitro. Infect Immun $\mathbf{6 2}$, 915-921.

Kojic, E. M. \& Darouiche, R. O. (2004). Candida infections of medical devices. Clin Microbiol Rev 17, 255-267.

Lo, H. J., Kohler, J. R., DiDomenico, B., Loebenberg, D., Cacciapuoti, A. \& Fink, G. R. (1997). Nonfilamentous C. albicans mutants are avirulent. Cell 90, 939-949.

Mathema, B., Cross, E., Dun, E. \& other authors (2001). Prevalence of vaginal colonization by drug-resistant Candida species in collegeage women with previous exposure to over-the-counter azole antifungals. Clin Infect Dis 33, E23-E27.

Naglik, J. R., Fidel, P. L., Jr \& Odds, F. C. (2008). Animal models of mucosal Candida infection. FEMS Microbiol Lett 283, 129139.

Nett, J., Lincoln, L., Marchillo, K. \& Andes, D. (2007a). Beta-1,3-glucan as a test for central venous catheter biofilm infection. J Infect Dis 195, 1705-1712.

Nett, J., Lincoln, L., Marchillo, K., Massey, R., Holoyda, K., Hoff, B., VanHandel, M. \& Andes, D. (2007b). Putative role of beta-1,3-glucans in Candida albicans biofilm resistance. Antimicrob Agents Chemother 51, 510-520.

NIH (1999). SBIR/STTR study and control of microbial biofilms. http://grants.nih.gov/grants/guide/pa-files/PA-99-084.html

Nobile, C. J. \& Mitchell, A. P. (2005). Regulation of cell-surface genes and biofilm formation by the C. albicans transcription factor Bcrlp. Curr Biol 15, 1150-1155.

Nobile, C. J., Andes, D. R., Nett, J. E., Smith, F. J., Yue, F., Phan, Q. T., Edwards, J. E., Filler, S. G. \& Mitchell, A. P. (2006a). Critical role of Bcrl-dependent adhesins in C. albicans biofilm formation in vitro and in vivo. PLoS Pathog 2, e63.

Nobile, C. J., Nett, J. E., Andes, D. R. \& Mitchell, A. P. (2006b). Function of Candida albicans adhesin Hwp1 in biofilm formation. Eukaryot Cell 5, 1604-1610.

Norice, C. T., Smith, F. J., Jr, Solis, N., Filler, S. G. \& Mitchell, A. P. (2007). Requirement for Candida albicans Sun 41 in biofilm formation and virulence. Eukaryot Cell 6, 2046-2055.

Owen, D. H. \& Katz, D. F. (1999). A vaginal fluid simulant. Contraception 59, 91-95.

Ramage, G., Vandewalle, K., Wickes, B. L. \& Lopez-Ribot, J. L. (2001). Characteristics of biofilm formation by Candida albicans. Rev Iberoam Micol 18, 163-170.

Ramage, G., VandeWalle, K., Lopez-Ribot, J. L. \& Wickes, B. L. (2002). The filamentation pathway controlled by the Efg1 regulator protein is required for normal biofilm formation and development in Candida albicans. FEMS Microbiol Lett 214, 95-100.

Ramage, G., Martinez, J. P. \& Lopez-Ribot, J. L. (2006). Candida biofilms on implanted biomaterials: a clinically significant problem. FEMS Yeast Res 6, 979-986.

Scott, T. G., Curran, B. \& Smyth, C. J. (1989). Electron microscopy of adhesive interactions between Gardnerella vaginalis and vaginal epithelial cells, McCoy cells and human red blood cells. J Gen Microbiol 135, 475-480. 
Slutsky, B., Buffo, J. \& Soll, D. R. (1985). High frequency switching of colony morphology in Candida albicans. Science 230, 666-669.

Sobel, J. D. (1992). Pathogenesis and treatment of recurrent vulvovagnial candidiasis. Clin Infect Dis 14, S148-S153.

Sobel, J. D., Faro, S., Foxman, B., Ledge, W. J., Nyirjesy, P. R., Reed, B. D. \& Summers, P. R. (1998). Vulvovaginal candidiasis: epidemiologic, diagnostic, and therapeutic considerations. Am J Obstet Gynecol 178, 203-211.

Stoldt, V. R., Sonneborn, A., Leuker, C. E. \& Ernst, J. F. (1997). Efglp, an essential regulator of morphogenesis of the human pathogen Candida albicans, is a member of a conserved class of bHLH proteins regulating morphogenetic processes in fungi. EMBO J 16, 19821991.

Swidsinski, A., Mendling, W., Loening-Baucke, V., Swidsinski, S., Dorffel, Y., Scholze, J., Lochs, H. \& Verstraelen, H. (2008). An adherent
Gardnerella vaginalis biofilm persists on the vaginal epithelium after standard therapy with oral metronidazole. Am J Obstet Gynecol 198, 97.

Taylor, B. N., Fichtenbaum, C., Saavedra, M., Slavinsky, I. J., Swoboda, R., Wozniak, K., Arribas, A., Powderly, W. \& Fidel, P. L., Jr (2000). In vivo virulence of Candida albicans isolates causing mucosal infections in people infected with the human immunodeficiency virus. J Infect Dis 182, 955-959.

van der Meijden, W. I., Koerten, H., van Mourik, W. \& de Bruijn, W. C. (1988). Descriptive light and electron microscopy of normal and cluecell-positive discharge. Gynecol Obstet Invest 25, 47-57.

Watamoto, T., Samaranayake, L. P., Jayatilake, J. A., Egusa, H., Yatani, H. \& Seneviratne, C. J. (2009). Effect of filamentation and mode of growth on antifungal susceptibility of Candida albicans. Int $J$ Antimicrob Agents 34, 333-339.

Edited by: K. Haynes 